\title{
GESTÃO DA INOVAÇÃO ABERTA NAS ORGANIZAÇÕES BRASILEIRAS
}

\author{
BRUNO HENRIQUES WATTÉ ${ }^{1}$; \\ MIGUEL ANGELO SILVEIRA ${ }^{2}$; \\ JOÃO ARTUR DE SOUZA ${ }^{3}$; \\ GERTRUDES DANDOLINI'; \\ PATRÍCIA DE SÁ FREIRE
}

\begin{abstract}
Open innovation has become one of the most important themes in innovation management. However, it is still unclear which management practices in the open innovation process in organizations are more effective. For this purpose, this research seeks, through an integrative literature review, to understand the current stage of development of the scientific literature about the best practices of open innovation management in Brazilian organizations. The presentation and discussion of the results was conducted based on four major thematic lines, that were common to most of the selected articles. As result, it is perceivable that the main benefit mentioned by the Brazilian organizations, is related to the reduction of product development costs, while the risks are shared.
\end{abstract}

Keywords: Open innovation; Open innovation practices; Open innovation processes.

Resumo: A inovação aberta tornou-se um dos temas mais importantes na gestão da inovação, entretanto ainda são obscuras as práticas de gestão mais eficazes no processo de inovação aberta nas organizações. Para isso a presente pesquisa busca por meio de uma revisão integrativa da literatura, entender o atual estágio de desenvolvimento da literatura científica sobre as melhores práticas de gestão de inovação aberta nas organizações brasileiras. A apresentação e discussão dos resultados do presente estudo foi conduzida a partir de quatro grandes linhas temáticas que foram comuns à maioria dos artigos selecionados.Sendo percebido que o principal benefício citado pelas organizações brasileiras, está relacionado a redução de custos de desenvolvimento de produtos, na medida em que os riscos são compartilhados.

Palavras-chave: Inovação aberta; Práticas de inovação aberta; Processos de inovação aberta.

\section{INTRODUÇÃO}

Poucos conceitos estiveram tanto em voga no meio empresarial nos últimos anos quanto a inovação. O recente crescimento e acirramento da competição entre as empresas, em

\footnotetext{
${ }^{1}$ Pós Graduação em Engenharia e Gestão do Conhecimento (UFSC) - Florianópois - Brasil: bruno@ watte.com.br ${ }^{2}$ Pós Graduação em Engenharia e Gestão do Conhecimento - (UFSC) - Florianópois - Brasil: miguel.pmsc@ gmail.com ${ }^{3}$ Pós Graduação em Engenharia e Gestão do Conhecimento - (UFSC) - Florianópois - Brasil: jartur@ gmail.com ${ }^{4}$ Pós Graduação em Engenharia e Gestão do Conhecimento - (UFSC) - Florianópois - Brasil: gertrudes.dandolini@ ufsc.br ${ }^{5}$ Pós Graduação em Engenharia e Gestão do Conhecimento - (UFSC) - Florianópois - Brasil: patriciadesafreire@ gmail.com
}

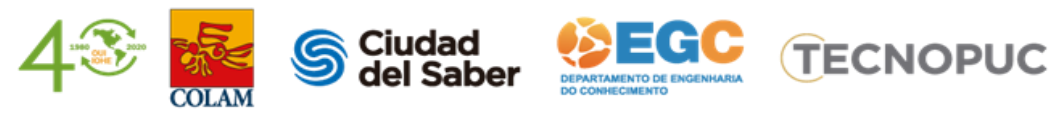


especial como decorrência dos processos de evolução e disseminação das tecnologias da informação e da globalização, e prinicpalmente no presente quadro de pandemia, decorrente do Covid-19, tornou a inovação praticamente uma obrigação para as empresas que objetivam se manter relevantes em seus mercados. Igartua et al.- (2010) afirmam que a inovação é um impulsionador chave de vantagem competitiva e do crescimento sustentável de negócios para as empresas.

Dentro desse contexto, um dos paradigmas mais amplamente discutido e implementado é a inovação aberta, termo cunhado por Chesbroughem 2003. Como referência da velocidade com que o interesse pela inovação aberta vem avançando, quando Huizingh et al.- (2011) publicaram seu artigo a respeito do estado da arte do constructo, em 2011, uma consulta ao termo "inovação aberta" no buscador do Google Acadêmico retornava cerca de 2 milhões de referências. Passados cerca de nove anos daquela publicação, em 2020, a mesma consulta aponta um total de publicações de 3,84 milhões, ou seja, praticamente o dobro.

Diante desse cenário, fica evidente que buscar aprofundar o entendimento a respeito dos avanços da pesquisa científica sobre as práticas de gestão mais eficazes no processo de inovação aberta nas organizações é um campo relevante de pesquisa e está em linha com as expectativas a respeito da evolução dos estudos sobre o constructo.

Nesse sentido o objetivo do presente estudo é entender o atual estágio de desenvolvimento da literatura científica sobre as melhores práticas da gestão de inovação aberta nas organizações brasileiras. Ou seja, se quer identificar o nível mais atualizado de desenvolvimento e de discussão a respeito desta temática. Alinhada a esse objetivo, a questão de pesquisa que norteará o artigo é: Qual é o estágio atual de desenvolvimento e de discussão científica a respeito das melhores práticas de gestão do processo de inovação aberta nas organizações brasileiras.

$\mathrm{Na}$ próxima seção abordaremos os fundamentos, conceituações sobre a inovação aberta e será abordado com mais profundidade as práticas em inovação aberta.

\section{REFERENCIAL TEÓRICO}

Um dos grandes desafios a respeito da inovação aberta, segundo Igartua et al.- (2010) é o cuidado redobrado necessário à gestão do processo de inovação aberta, em especial diante 
da complexidade introduzida pela ampla interdependência entre organizações. Segundo eles, muitos estudos concentraram energia no entendimento das medidas objetivas de entradas e saídas do processo de inovação, porém ignorando o processo intermediário de práticas de gestão da inovação aberta. Huizingh et al.-(2011) reforçam que o melhor entendimento das práticas da inovação aberta (como fazer) é um dos grandes campos de pesquisa a respeito do tema, uma vez que ela requer dos gestores novas decisões durante o desenvolvimento e exploração de atividades de inovação. Segundo eles, a gestão do processo de inovação aberta ainda acontece basicamente através de um método de tentativa e erro faltando pesquisas mais aprofundadas que levem a um framework que efetivamente ajude os gestores nas decisões sobre quando e como empregar cada uma das práticas da inovação aberta. Gassmann et al.(2010) concordam e apontam pesquisas a respeito dos processos de inovação aberta como uma das tendências mais atuais das pesquisas sobre inovação.

\subsection{INOVAÇÃO ABERTA}

Inovação aberta pode ser definida como sendo “(...) o uso intencional de fluxos de entrada e de saída de conhecimento na organização, para acelerar a inovação interna, e expandir mercados para o uso externo da inovação, respectivamente" (Chesbrough et al.2006). Huizingh et al.- (2011) argumentam que, na prática, nem o uso de opiniões externas para melhorar o processo interno de inovação e nem a busca de oportunidades de comercialização das inovações fora da empresa são essencialmente novos. Para eles, o que tornou o constructo proposto por Chesbrough et al.- (2006) tão atrativo para acadêmicos e praticantes foi uma combinação entre o fato de ser um conceito "guarda-chuva", associado ao timing adequado de proposição, à ampla oportunidade de extensão e desenvolvimento do constructo e à conexão entre fluxos de entrada e saída de conhecimento nas organizações, sendo que a entrada e saída de conhecimento pode usar várias fontes, inclusive fontes de ideias externas.

Inovação aberta tornou-se um dos temas mais quentes na gestão da inovação. O processo de Inovação Aberta refere-se a tanto a transição para a Inovação Aberta como as suas várias práticas de Inovação Aberta. Dois processos de inovação aberta são relevantes. Em primeiro lugar, o processo que leva à inovação aberta, é o processo de práticas de 
inovação de abertura que anteriormente eram (mais) fechados. O segundo processo refere-se às práticas de como fazer a inovação aberta.

O processo de transição de fechado para aberto detalha os passos através do qual firmas abrem o seu processo de inovação. De acordo com Gassmannet al.- (2010) inovação aberta muitas vezes começa com a terceirização de contratar organizações de serviço, a ser seguido por modos mais estratégicas de inovação aberta.

O segundo processo de inovação aberta está relacionado com a questão de "como fazêlo?”. A inovação aberta exige que os gestores para tomar novas decisões no desenvolvimento e exploração de atividades de inovação, devendo saber: Quando, como, com quem, com que propósito, e de que maneira que eles devem cooperar com terceiros? Os envolvidos variam de fornecedores, clientes e concorrentes, a instituições e organizações de pesquisa em diferentes indústrias que ou têm soluções que podem melhorar as inovações da empresa ou que podem explorar soluções a empresa tem desenvolvido. Estas colaborações podem durar por um período de significativa, são susceptíveis de ser repetido, envolvem diferentes grupos de organizações, pode ter diferentes iniciadores, requerem diferentes papéis da organização, e incluem diferentes departamentos. (Huizinghet al.- 2011). Conforme o modelo de processo e de mercado poderá ser construído o modelo de negócio em inovação aberta. Uma tecnologia medíocre seguida de um grande modelo de negócio, poderá ser mais valiosa do que uma tecnologia fantástica usada num modelo de negócio medíocre. (Chesbrohgh, 2012, p.81).

\subsection{PRÁTICAS EM INOVAÇÃO ABERTA}

Sobre as práticas em inovação aberta Fetterhoff e Voelkel (2006) propõem um modelo de "como fazer inovação aberta" que inclui as seguintes cinco etapas: 1) busca de oportunidades, 2) avaliação de seu potencial de mercado e inventividade, 3) recrutamento de potenciais parceiros de desenvolvimento, 4) captura de valor através da comercialização e 5) ampliando a oferta de inovação.

Nessa mesma linha, Igartua et al.-(2010) apontam que a prática da inovação aberta se apoia em treze componentes centrais: Estratégia da Inovação (o que, porque, quando e metas); Gestão de Portfólio (escolhas, foco e necessidades); Gestão de Projeto (complexo devido a risco e incerteza); Liderança e Cultura (Promoção, Suporte e Comunicação); Recrutamento 
(capacidade de atração e retenção talentos); Relações Externas (nutrição de redes, processos de troca); Design Organizacional (incorporação e aplicação da inovação); Processos / Framework de Inovação (metodologias); Mensuração de Performance (sistema adequado, medir certo); Marketing (geração de ideias adequadas); Recursos (tempo, pessoas e capital); Gestão da Propriedade Intelectual; Tecnologia.

\section{PROCEDIMENTOS METODOLÓGICOS}

A metodologia de pesquisa utilizada no presente estudo foi a revisão integrativa, baseada na definição proposta por Broome (apudWhittemore \&Knafl, 2005): "um método específico de revisão que sumariza a literatura teórica e empírica passada, de modo a prover um entendimento mais compreensivo de um fenômeno particular". Pois, a revisão integrativa produzir uma síntese sobre o passado da pesquisa a respeito do tema em estudo, que pode se tornar compreensiva e apontar o estado da arte a respeito do fenômeno, desde que realizada com o rigor adequado.

Quando desenvolvida baseada em adequado nível de rigor metodológico, a revisão integrativa se torna também reprodutível. Ou seja, caso os passos metodológicos previstos sejam seguidos adequadamente, dois pesquisadores distintos serão capazes de chegar a resultados semelhantes ao realizar a pesquisa sobre o constructo. Para se chegar a esse resultado, Botelho, Cunha e Macedo (2011) propõem uma sequência de seis etapas a saber: 1) identificação do tema e seleção da questão de pesquisa; 2) estabelecimento dos critérios de inclusão e exclusão; 3) identificação dos estudos pré-selecionados e selecionados; 4) categorização dos estudos selecionados; 5) análise e interpretação dos resultados; 6) apresentação da revisão/síntese do conhecimento.

Para alcance dos objetivos propostos no presente artigo, as buscas foram concentradas nas bases de dados Scopus, Scielo e Web of Sciencebuscando reunir, após uma de avaliação inicial dos artigos obtidos e da aplicação dos critérios de exclusão, cerca de 20 artigos dentre os mais citados.

Para o processo de definição das palavras-chave utilizadas na busca, realizou-se uma pesquisa prévia no motor de buscas do Google Acadêmico, de modo a identificar os temos que mais frequentemente estavam associados à prática de Inovação Abertanas organizações.

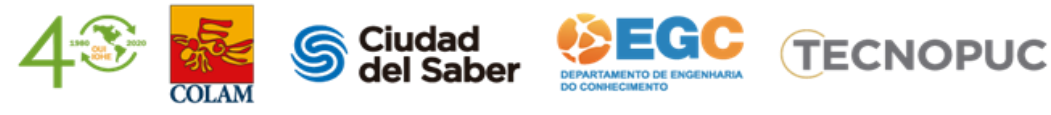


Essa pesquisa apontou duas palavras-chave centrais: "management" e "practice". Uma vez que o interesse do estudo estava centrado em casos de organizações brasileiras, a string de busca utilizada foi: "inovação aberta" and brazil and ("management" or "practice”)

Dado o objetivo de identificar o nível mais atualizado de desenvolvimento e de discussão a respeito desta temática nas organizações brasileiras, optou-se por restringir a busca apenas a artigos publicados nos últimos cinco anos. Adicionalmente, do ponto de vista do tipo de publicação, optou-se por restringir o estudo a texto publicados em "journals" ou em anais de congressos internacionais, adicionando aos critérios de exclusão os livros completos, capítulos de livros, ou produtos tecnológicos. Essa exclusão baseou-se na necessidade de obtenção de textos com o devido rigor científico, uma vez que são textos avaliados por pares.

A busca realizada nas bases de dados, a partir das palavras-chave selecionadas, resultou numa lista inicial de quarenta e nove publicações. Após a exclusão dos artigos anteriores a 2015 e dos artigos duplicados nas diferentes bases (eram sete) a lista foi reduzida a 30 publicações, sendo 28 artigos científicos e duas publicações de outros tipos. A forte concentração de publicações realizadas nos cinco últimos, deixa evidente a recência da pesquisa sobre a prática deinovação aberta.

Esses vinte e oitos artigos foram ordenados segundo o critério do maior número de citações e formaram a lista de artigos pré-selecionados. Dessa relação foram avaliados individualmente os títulos, as palavras-chave e o resumo, de modo a identificar aqueles que efetivamente estavam relacionados à prática de inovação abertaem organizações. Feita a exclusão daqueles artigos desconectados do objetivo do presente estudo, restaram dezoitos artigos selecionados para a leitura na íntegra, em seguida três artigos foram excluídos por não possuírem relação com o estudo desta pesquisa, sendo reduzidos para quinze textos finais, incluídos no presente estudo após esta leitura completa.

Uma breve análise inicial dos quinze textos finais permitiu a identificação de algumas características interessantes a seu respeito. Apenas dois artigos eram de pesquisa de base teórica e trezes estavam baseados em estudos empíricos. Dos trezes estudos empíricos, oito eram de casos relacionados ao setor público, três ao setor privado e dois tratavam da relação entre o público e o privado. Vale destacar que todos os trezes estudos empíricos estavam 
baseados em metodologias qualitativas, alinhadas à objetivos de natureza mais exploratória ou de entendimento em profundidade do assunto.

Quanto ao ano de publicação, praticamente a metade dos artigos (sete dos quinze finais) foram publicadasentre 2018 e 2019, o que reforça o crescente interesse científico a respeito do tema. Quanto à indústria ou setor da economia pesquisado observa-se uma grande dispersão, havendo estudos nas áreas da educação, construção civil, automotiva, aeronáutica, química, saúde, tecnologia e agropecuária, dentre outros. A diversidade de áreas denota um interesse generalizado pelo tema de inovação aberta.

A matriz de síntese foi utilizada para garantir a eficácia desejada para o processo de categorização e análise dos textos. Segundo Botelho, Cunha e Macedo (2011) a matriz de síntese tem por objetivo "a extração e organização dos dados de revisão da literatura... e proteger o pesquisador de erros durante a análise". Segundo os autores, não existe uma matriz certa ou errada, mas sim a matriz que permite a cada pesquisador realizar o melhor processo de categorização e análise das informações. A matriz de síntese desenvolvida no presente estudo reuniu as seguintes informações de cada um dos artigos: gap de pesquisa, objetivo ou questão de pesquisa, método de pesquisa, setor econômico, tipo de indústria, requisitos para a prática de Inovação Aberta, práticas de Inovação Aberta, barreiras ou desafios à prática de Inovação Aberta, relação entre Inovação Abertae inovação e sugestão de pesquisa futura.

\section{RESULTADOS}

Um dos artigos teóricos analisados, desenvolvido por De Paulo et al.-.(2017), apresenta uma revisão bibliométrica a respeito do constructo inovação aberta, comparando as publicações realizadas nos países desenvolvidos (participantes do G7 - Alemanha, Canadá, Estados Unidos, França, Itália, Japão e Reino Unido) com aquelas realizada nos países em desenvolvimento (Brasil, Índia, China, Rússia e África do Sul), sujeitos a condições econômicas e sociais desfavoráveis. Confirmando a expectativa inicial dos autores, no período analisado (2000-2014) o volume de publicações sobre inovação abertanos países em desenvolvimento é substancialmente inferior àquele dos países desenvolvidos (63\% menor). Mas quando o critério de comparação deixa de ser o volume de publicações e passa para o número de citações aos artigos (um proxy da qualidade dos estudos), a diferença se aprofunda 
significativamente. O volume de citações dos países em desenvolvimento é $98 \%$ menor do que aqueles do G7.

Ao se avaliar a qualidade dos artigos listados no presente estudo, utilizando-se como proxy para a isso a avaliação Qualis/Capes das revistas em que foram publicados, pode se chegar a uma constatação semelhante à do estudo bibliométrico citado anteriormente. Nenhum dos artigos selecionados foi publicado em uma revista classificada como "A" pela Qualis/Capes. Dos quinze artigos, cinco foram publicados em revista sem conceito algum, seis foram publicados em revista com classificação "B1", um deles em revista "B2", dois em revista "B3" e um em revista "B4". A análise do volume de citações, realizada através do "Google Acadêmico" para todos os artigos, revela um cenário semelhante: mais da metade dos artigos selecionados (nove de quinze) não possui nenhuma citação. Dos seis artigos que receberam alguma citação, dois eles tiveram uma única, um deles teve duas citações, dois tiveram seis citações e um dos artigos teve catorze citações.

Ainda a respeito do conjunto dos artigos selecionados, é interessante notar que não existe uma uniformidade na relação conceitual que eles constroem entre o constructo inovação abertae a definição mais tradicional de inovação. um total de nove dentre os quinze artigos, trata dessa relação. quatro autores (boas et al., 2017; Erlaine et al., 2017; Candido e Do Vale, 2018; de Paulo et al., 2017) tratam inovação abertacom uma nova abordagem ou um novo paradigma da gestão da inovação, que quebra com o entendimento convencional e linear da abordagem tradicional, em favor de um entendimento mais complexo e interativo, baseado em aprendizado. três deles (Leme et al., 2015; Moraes, de Oliveira e Miranda, 2016; da Silva et al., 2016) tratam inovação abertacomo a atual geração do processo de inovação nas organizações, que veio passando por uma sequencia de etapas de evolução ao longo do tempo até chegar neste estágio atual. dos outros dois autores que tratam dessa relação, um deles descreve inovação abertacomo uma prática da gestão da inovação (Ferrari, Scaliza e Jugend, 2019) e outro entende que inovação fechada e inovação abertasão práticas completamente distintas, que podem inclusive conviver simultaneamente dentro das organizações (Thomas, 2018).

A sequência da apresentação e discussão dos resultados do presente estudo se conduzirá a partir de quatro linhas temáticas que foram comuns à maioria dos artigos 
selecionados, a partir de uma leitura transversal de todos eles, sendo:1) os benefícios da abordagem de inovação aberta; 2) os requisitos ou pré-condições para o processo de inovação abertapossa apresentar resultados satisfatórios; 3) as práticas efetivas de inovação abertae 4) as barreiras ou dificuldades relacionadas à um processo de inovação aberta.

Sobre os benefícios de inovação aberta, cerca de 50\% dos artigos (oito dentre os quinze) tratou explicitamente dos benefícios que a implementação do conceito de inovação abertaé capaz de trazer para as organizações. O benefício mais citado com a implementação dessa prática (quatro dos oito artigos) é a redução de custos no desenvolvimento de novos produtos (Candido e do Vale, 2018; Macedo-Soares et al., 2017; Leme et al., 2015; Erlaine et al., 2017), que pode ser vista também como uma espécie de compartilhamento dos riscos de pesquisa e desenvolvimento (Leme et al., 2015).Além desse ponto relativo à redução de custos, alguns autores abordam especificamente as possibilidades e as oportunidades vinculadas a novos fluxos de conhecimento para a área de pesquisa e desenvolvimento, numa visão de processo outside-in da inovação aberta. Leme et al.-(2015) destacam que, no contexto de um instituto público de pesquisa, a prática acelera o aprendizado e possibilita a transferência externa de conhecimento. Por fim, Scherer e Ribeiro (2015), concluem que a utilização da prática de crowdsourcingpor meio de portais de ideias eleva significativamente a quantidade de ideias geradas a respeito de novos produtos e acelera os processos de teste e refino dessas ideias.

Sobre os requisitos para inovação aberta, há vários autores selecionados na pesquisa concordam que nem todas as organizações conseguem se beneficiar de maneira satisfatória da implementação da inovação aberta. Isso acontece porque alguns processos internos à organização ou vinculados à sua relação com os parceiros precisam estar adequadamente preparados ou desenvolvidos para que os fluxos de conhecimentos viabilizados pela inovação aberta possam proporcionar os benefícios esperados.Macedo-Soares et al.-(2017) tratam dessa questão a partir de um paradigma de análise de redes. Segundo eles, do ponto de vista das relações de parceria, a posição relativa da organização na rede é uma importante variável na análise dos benefícios proporcionados pela inovação aberta. Candido e do Vale (2018), num estudo a respeito de um polo tecnológico de Florianópolis, e Leme et al.-(2015), num estudo centrado no Instituto Butantã em São Paulo, reforçam a importância da preparação da

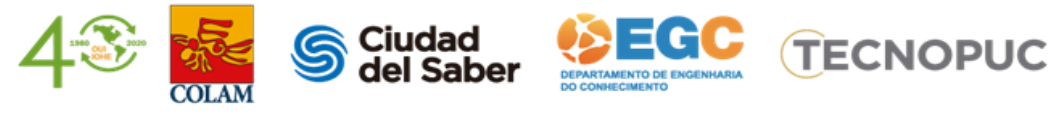


estrutura organizacional interna para que a empresa possa maximizar os benefícios das práticas de inovação aberta. Leme et al.- (2015) usam como base de avaliação um framework proposto por Chiaroni, Chiesa e Frattini (2011) e que propõem quatro grandes bases de gestão para as práticas de inovação aberta: 1) construção e operação da rede de parceiros, 2) estrutura organizacional interna, 3) avaliação das necessidades e fluxos de conhecimento, 4) gestão do conhecimento. Ao final do artigo Leme et al.- (2015) apontam que o desenvolvimento da rede de parceiros não deveria ser o primeiro passo das organizações na implementação de processos de inovação aberta, pois os avanços na interação com os parceiros demandam estruturas internas capazes de integrar esta rede e os fluxos de conhecimento gerados.

Alguns outros autores centram sua abordagem relativa aos requisitos para a inovação aberta na importância dos processos de comunicação, tanto internos quanto externos. Erlaine et al.- (2017) citam que uma atitude organizacional aberta é crucial para o sucesso da prática da inovação aberta, uma vez que esta prática tem impacto direto nas atividades de comunicação e relações públicas das empresas. Por fim, Scherer e Ribeiro (2015) destacam o papel que a internet e as novas ferramentas de comunicação têm na facilitação e intensificação deste processo de comunicação entre os parceiros na rede.

Sobre as barreiras e dificuldades de inovação aberta mencionadas nos artigos selecionados estão relacionadas, essencialmente, a quatro fatores centrais: identificação dos parceiros adequados(Ferrari;Scaliza; Jugend, 2019; Paulo et al., 2017), gestão da relação com os parceiros (de Souza Andrade et al., 2016), gestão interna da inovação aberta (Leme et al., 2015;Erlaine et al., 2017) e custos incrementais decorrentes da maior complexidade de gestão (Ferrari;Scaliza;Jugend, 2019; Scherer; Ribeiro, 2015).Ferrari, Scaliza e Jugend (2019) mencionam que a identificação do(s) parceiro(s) adequados é a primeira das dificuldades associadas à implantação da inovação aberta nas organizações. De Paulo et al.- (2017) concordam, e reforçam que devido a crescentes diferenças de competências e cultura entre nações e indústrias, as abordagens tradicionais para identificação de parceiros já não são suficientes. Rocha, Mamédio e Quandt (2019) citam algumas das variáveis centrais a se levar em consideração na identificação do(s) parceiro(s) adequado(s): existência de diferenças culturais, práticas de processos ágeis vs. processos estáticos, diferenças entre éticas de trabalho e na disposição para assumir riscos. 
No que se refere à gestão da relação com parceiros, De Souza Andrade et al.- (2016), analisando o caso de implementação da inovação aberta no ITA (Instituto Tecnológico da Aeronáutica) destacam que uma das importantes dificuldades a serem superadas está relacionada a burocracias e questões regulatórias decorrentes da relação entre o público e o privado. Avaliando essa mesma questão sob o prisma da relação entre startups e empresas estabelecidas, Rocha, Mamédio e Quandt (2019) destacam que as diferenças de culturas geracionais e a eventual diferença de competências sobre novas tecnologias podem ser barreiras na relação entre parceiros de uma rede de inovação aberta. Candido e do Vale (2017) destacam ainda a importância da construção de uma relação de confiança entre parceiros, para que se possa obter o máximo benefício da inovação aberta.

Em relação à gestão interna de inovação aberta, Leme et al.- (2015) citam que a principal barreira é a necessidade de mudança de estruturas e processos internos nas organizações participantes. Erlaine et al.- (2017) concordam e destacam que a organização precisa dispor de recursos e competências específicas, além de um ambiente institucional favorável. Ainda nessa linha, Thomas (2018) destaca que a cultura organizacional dos países em desenvolvimento não valoriza adequadamente as práticas de proteção intelectual, o que é uma barreira a ser contornada nas organizações presentes nesses países.

Por fim, alguns autores citam a questão dos custos incrementais para se gerir os processos de inovação aberta. Segundo Ferrari, Scaliza e Jugend (2019) esses custos podem ser vinculados à detecção de fluxos externos de conhecimento mais adequados, à assimilação ou à utilização desse conhecimento. Scherer e Ribeiro (2015) adicionam a questão de que os prazos de desenvolvimento de novos produtos podem ser maiores nas práticas de inovação aberta, quando comparados às práticas tradicionais ou fechadas de inovação. Eles citam ainda que parte desses prazos mais dilatados pode estar relacionado à objetivos distintos entre os parceiros.

Quadro 01 - Barreiras

\begin{tabular}{|c|c|}
\hline Barreiras & Fontes \\
\hline Identificação dos parceiros adequados & Ferrari; Scaliza; Jugend, 2019; paulo et al., 2017 \\
\hline Gestão da relação com os parceiros & De Souza Andrade et al., 2016 \\
\hline Gestão interna da inovação aberta & Leme et al., 2015; Erlaine et al., 2017 \\
\hline
\end{tabular}


Fonte: Dos Autores

Sobre as práticas de inovação aberta, a última das grandes linhas temáticas observadas, diz respeito à identificação das principais práticas de inovação aberta presentes nos artigos selecionados. Vale notar que essa identificação se dá a partir de uma pluralidade de contextos uma vez que, como mencionado anteriormente, os quinze artigos abordam diferentes indústrias e setores.

Nos artigos que tratam das práticas da inovação aberta no contexto do setor público é possível perceber forte preocupação com a dimensão legal ou regulatória dessa prática. Tanto De Souza Andrade et al.-(2016), tratando do caso do ITA, quanto Leme et al.-(2015), tratando do caso do Instituto Butantã, dão grande relevância à temática da propriedade intellectual. Essapreocupação com a propriedade intelectual não se observa nas grandes empresas privadas. Thomas (2018) evidencia claramente esse aspecto, apontando que nenhuma das duas empresas do setor químico estudadas por ela dá importância para o assunto. Segundo a autora, trata-se de um traço cultural das empresas privadas brasileiras. Nos artigos selecionados, o que a organizações do setor privado brasileiro mais valorizam e praticam, em se tratando de inovação aberta, são os processos outside-in, de fluxos externos de conhecimento alimentando os ciclos de pesquisa e desenvolvimento de novos produtos. Nessa linha, Scherer e Ribeiro (2015) trazem casos de três grandes empresas que usam portais na internet como fonte de relacionamento com parceiros e estimulação de fluxos externos de conhecimento para dentro das organizações. Darrigo e Fachinelli (2015) fazem uma análise semelhante, mas mais detalhada, de uma única grande empresa.

Erlaine et al.-(2017), analisando a cadeia produtiva do agronegócio alimentício constatam que nessa cadeia o estímulo à adoção da inovação aberta é top-down, definido pelo elo central da rede (normalmente a maior empresa), que também coordena todo o processo. Segundo eles, é muito baixa a interatividade das empresas privadas brasileiras com as universidades, com o que concorda Thomas (2018).

Por fim, o grande traço que marca a prática da inovação aberta nas startups e pequenas empresas privadas é a informalidade. Rocha, Mamédio e Quandt (2019), analisando 4 startups 
de um polo tecnológico em Minas Gerais apontam quatro aspectos que ilustram esta informalidade: falta de critério para definir a colaboração com outras empresas, ausência de indicadores de performance para avaliar a efetividade dos processos, troca de informações, ideias e habilidades ocorrendo essencialmente através de conversas pessoais ou email e poucas ações planejadas. Candido e do Vale (2018) avaliando empresas de um polo tecnológico em Florianópolis apontam, da mesma forma, a existência de redes informais ou processos informais de networking, como sendo uma prática marcante de inovação aberta das startups avaliadas. Moraes, De Oliveira e Miranda (2016), estudando sete pequenas empresas de base tecnológica do Vale do Paraíba, em São Paulo, reforçam esse ponto, apontando a necessidade de maior formalização dos processos através de patentes ou modelos utilitários como sendo próximos passos da evolução da atuação daquelas empresas no contexto da inovação aberta.

\section{CONSIDERAÇÕES FINAIS}

O tema da inovação está na agenda de prioridades da maioria das organizações, de todos os tamanhos, nos diversos setores e indústrias. $\mathrm{O}$ recente salto na velocidade e na dimensão do desenvolvimento de novas tecnologias está na base dessa agenda de inovação, na medida em ele alimenta grandes mudanças nos hábitos dos consumidores e viabiliza uma intensificação do processo competitivo entre as empresas. Nesse cenário, o paradigma da inovação aberta começa a crescer. A inovação aberta é aquela que não ocorre exclusivamente dentro das fronteiras das organizações. Ao contrário, se beneficia das redes de relacionamento formais e informais mantidas por elas. Dado o atual nível de interconectividade experimentado pelos indivíduos e pelas empresas, há cerca de 8 anos atrás, sabia-se que não seria possível se praticar outro tipo de inovação que não fosse a inovação aberta.

Neste contexto, o presente estudo objetivou entender um pouco mais profundamente a realidade das organizações brasileiras quanto às suas práticas de gestão da inovação aberta, se propondo a responder à seguinte questão de pesquisa: Qual é o atual estágio de desenvolvimento e de discussão científica a respeito das práticas de gestão do processo de inovação aberta nas organizações brasileiras? 
Para respondê-la, o estudo baseou-se em uma revisão integrativarealizada nas bases de dados Scopus, Scielo e WebOfScience e foram selecionados, ao final da pesquisa, quinze artigos publicados nos últimos cinco anos.

Ainda que a qualidade dos artigos selecionados não esteja alinhada aos melhores artigos sobre o tema publicados nos países desenvolvidos, foi possível identificar entre eles quatro grandes linhas temáticas que foram apresentadas e discutidas: os benefícios da inovação para as organizações, os requisitos para sua implementação, as barreiras e dificuldades associadas à essa prática e as principais atividades práticas de inovação aberta identificadas nos artigos. De uma maneira geral, o principal benefício citado pelas organizações brasileiras, está relacionado a redução de custos de desenvolvimento de produtos, na medida em que os riscos são compartilhados.

Em relação aos requisitos, a principal leitura é de que uma reestruturação interna na organização para gerir os processos de inovação aberta precisa anteceder a formação das redes de parceiros. A identificação dos parceiros certos e a gestão desta relação são as principais dificuldades associadas a inovação aberta. Já em relação às principais práticas identificadas, elas variam conforme o contexto: nas empresas públicas está relaciona à gestão da propriedade intelectual, nas grandes empresas privadas está centrada na captura de conhecimento externo a partir de fluxos outside-in e nas startups e pequenas empresas as práticas da inovação aberta são marcadas por grande informalidade.

Apesar de ter atingido seu objetivo, o presente estudo apresenta algumas limitações importantes. A principal delas está relacionada ao fato de terem sido identificados apenas quinze artigos e de serem, em sua maioria de qualidade abaixo da necessária para que seja possível estender as conclusões aqui apresentadas a um universo mais amplo de empresa.

\section{REFERÊNCIAS}

Boas, Karen Moreira Vilas et al.- Platforms of Ideas Management and Open Inovation: The Crowdstorm Approach Applied to Public University in Brazil. In: International Conference on Electronic Government and the Information Systems Perspective. Springer, Cham, 2018. p. 205-217.

Botelho, L.; Cunha, C.; Mavedo, M. O método da revisão integrativa nos estudos organizacionais. Gestão e sociedade, v. 5, n. 11, p. 121-136, 2011. 
Cândido, Ana Clara; Do Vale, Mariene Alves. Práticas de gestão da informação e inovação aberta: estudo no pólo tecnológico de Florianópolis. Perspectivas em Ciência da Informação, v. 23, n. 4, p. 184-204, 2018.

Chesbrough, H.; Vanhaverbeke, W.; West, J. (Ed.). Inovação Aberta: Researching a new paradigm. Oxford University Press on Demand, 2006.

Chiaroni, Davide; Chinesa, Vittorio; Franttini, Federico. The Open Innovation Journey: How firms dynamically implement the emerging innovation management paradigm. Technovation, v. 31, n. 1, p. 34-43, 2011.

Da Silva, Davi N. et al.- Optimización del Proceso de Innovación para Proyectos Internos en las Empresas. Información tecnológica, v. 27, n. 3, p. 119-130, 2016.

Darrigo, Fernanda; Fachinelli, Ana Cristina. Crowdsourcing and Open Inovation for sustainable company growth: a Brazilian case study. 2015.

De Paulo, Alex Fabianne et al.- Mapping Open Inovation: A Bibliometric review to compare developed and emerging countries. Global Business Review, v. 18, n. 2, p. 291-307, 2017.

De Souza Andrade, Herlandí et al.- The management of Intellectual Property in the Technology License Office in an Open Inovation environment: The context of a Scientific and Technological Institution in Brazil. In: 2016 Portland International Conference on Management of Engineering and Technology (PICMET). IEEE, 2016. p. $1630-1637$.

Erlaine, Binotto et al.- Evidences of Open Inovation in Brazilian Agrifood Chain. Revista ESPACIOS, v. 38, n. 28, 2017.

Ferrari, Aline Gabriela; Scaliza, Janaina Aparecida Alves; JUGEND, Daniel. The landscape of Open Inovation in Brazil: An analysis of the recent literature. Production, v. 29, 2019.

Gassmann, O.; Enkel, E.; Chesbrough, H.. The future of Open Inovation. R\&d Management, v. 40, n. 3, p. 213-221, 2010.

Huizingh, E. Kre. Open Inovation: State of the art and future perspectives. Technovation, v. 31, n. 1, p. 2-9, 2011.

Igartua, J.I.; Garrigós, J.A.; Hervas-Oliver, J.L. How innovation management techniques support an Open Inovation strategy. Research-Technology Management, v. 53, n. 3, p. 41-52, 2010.

Leme, Ana PFP et al.- The implementation of Open Inovation: A case study of managerial levels in a centenary public research institute. In: 2015 Portland International Conference on Management of Engineering and Technology (PICMET). IEEE, 2015. p. 736-746.

Macedo-Soares, T. Diana L. et al.- Leveraging firm innovation performance through alliance portfolios in emerging economies: the role of absorptive capacity. Journal of technology management \& innovation, v. 12, n. 4, p. 10-21, 2017.

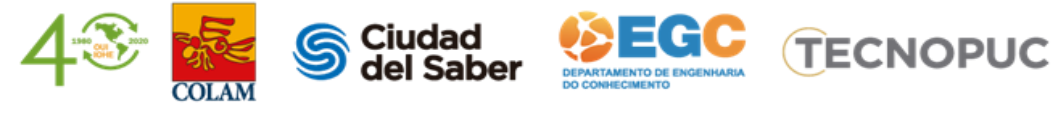


Moraes, M. B.; De Oliveira, E. A. A. Q.; Miranda, M. A. S. Proposal for an innovation model for Brazilian small and medium technology-based enterprises. In: IAMOT 2016-25th International Association for Management of Technology Conference, Proceedings: Technology-Future Thinking. International Association for Management of Technology Conference (IAMOT) and the Graduate School of Technology Management, University of Pretoria, 2016.

Rocha, Clarissa Figueredo; Mamédio, Diórgenes Falcão; Quandt, Carlos Olavo. Startups and the innovation ecosystem in Industry 4.0. Technology Analysis \& Strategic Management, p. 1-14, 2019.

Scherer, Jonatas Ost; Ribeiro, José Luis Duarte. Identifying important features in the scope of an Open Inovation project via a web portal. International Journal of Business Innovation and Research, v. 9, n. 2, p. 133-147, 2015.

Thomas, Elisa. From closed to Open Inovation in emerging economies: Evidence from the chemical industry in Brazil. 2018.

Vieira, Filipe C.; Vale, Hamilcar V.; May, Marcia R. Inovação Aberta and Business Model: Embrapaforestry case study. RAM. Revista de Administração Mackenzie, v. 19, n. 4, 2018.

Whittemore, R.; Knafl, K. The integrative review: updated methodology. Journal of advanced nursing, v. 52, n. 5, p. 546-553, 2005. 